Rechtsmedizin 2010 - 20:249-249

DOI 10.1007/s00194-010-0697-x

Online publiziert: 11 . Juli 2010

(c) Springer-Verlag 2010

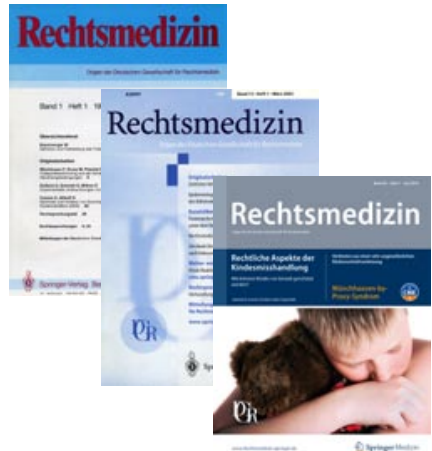

Im September 1990 hat die Mitgliederversammlung der Deutschen Gesellschaft für Rechtsmedizin (DGRM) in ihrer Satzung festgelegt: „Publikationsorgan ist die Zeitschrift Rechtsmedizin“. - Dies war ein Neustart nach einer seinerzeit langwierigen und $\mathrm{z}$. T. durchaus kontroversen Diskussion. Dabei ging es um das Konzept einer in Deutschland herausgegebenen internationalen Fachzeitschrift in Fortführung der traditionsreichen Zeitschrift für Rechtsmedizin (seit 1991 International Journal of Legal Medicine). Wir haben die wechselvolle Entwicklung im Zeitschriftenwesen der DGRM anlässlich der 100-Jahr-Feier ausführlich dargelegt [1].

Die Rolle als Organ der DGRM übernahm nunmehr die neu gegründete Zeitschrift Rechtsmedizin. Den Spagat zwischen der internationalen Ausrichtung (in englischer Sprache) einerseits sowie der Pflege traditioneller Stärken innerhalb der DGRM andererseits wurde dadurch erleichtert, dass die jeweiligen geschäftsführenden Herausgeber auch zu den Mitherausgebern der anderen Zeitschrift gehören. Erster geschäftsführender Herausgeber der neuen Zeitschrift Rechtsmedizin wurde M. Staak (Köln). Mit Band 10 (1999/200o) fand der erste Wechsel statt, und G. Geserick (Berlin) übernahm diese Position dann bis einschließlich Band 12 (2002). Seit Band 13 (2003) liegt diese ehrenvolle Aufgabe bei K. Püschel (Hamburg).

M. Staak hat die neu gegründete Zeitschrift von Anfang an mit ruhiger Hand

\author{
K. Püschel ${ }^{1} \cdot$ M. Kretz ${ }^{2} \cdot$ D. Lorenz ${ }^{2}$ \\ ${ }^{1}$ Institut für Rechtsmedizin, Universitätsklinikum Hamburg-Eppendorf, Hamburg \\ ${ }^{2}$ Springer-Verlag $\mathrm{GmbH}$, Heidelberg
}

\title{
Zwei Jahrzehnte Rechtsmedizin
}

sicher durch die nicht ganz unkomplizierten ersten Erscheinungsjahre geführt. Diese Mission war schwierig, aber sie wurde gut gemeistert.

\section{- Rechtsmedizin hat sich nicht nur im deutschsprachigen Raum überzeugend positioniert, sondern sie findet zunehmend auch internationale Beachtung (z. B. durch die ausführlichen englischen Abstracts).}

Das ursprüngliche Konzept der Zeitschrift (z. B. mit den ständigen Rubriken Rechtsprechungsteil, Mitteilung der DGRM, Buchbesprechungen) wurde behutsam weiterentwickelt (z. B. mit CME Weiterbildung - Zertifizierte Fortbildung, Leitthemenheften). Auch die äußere Erscheinungsweise wurde mehrfach modern umgestaltet. Durch den angemessenen wissenschaftlichen Anspruch ist auch die Entwicklung bei den Manuskripteinsendungen positiv. Die seit Langem angestrebte Listung der Zeitschrift ist in Sicht; ab Mitte nächsten Jahres wird die Zeitschrift Rechtsmedizin über einen „Impact“-Faktor verfügen. Und im Hinblick auf die weitere $\mathrm{Zu}$ kunft gibt es eine sehr vertrauensvolle gemeinsame Perspektive zwischen der Fachgesellschaft und dem Verlag.

Wir freuen uns alle (DGRM, Herausgeber und Verlag) sehr darauf, das 20-jährige Jubiläum unserer Zeitschrift Rechtsmedizin anlässlich der 89. Jahres-tagung der DGRM in Berlin zu begehen. - In den nächsten Heften von Rechtsmedizin wird dann die Gelegenheit bestehen, hierüber ausführlicher zu berichten und eine Bilanz der ersten zwei Jahrzehnte zu ziehen. Nach 20 Jahren erscheint die Rechtsmedizin mit einem sehr eigenständigen Charakter nachhaltig gefestigt und blickt positiv in die Zukunft.

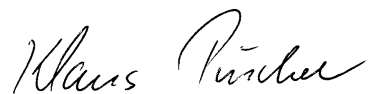

Prof. Dr. K. Püschel

Geschäftsführender Herausgeber

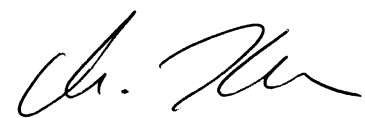

Monika Kretz

Stellv. Leitung Fachzeitschriften Medizin/ Psychologie

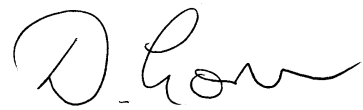

Dagmar Lorenz

Redaktion Rechtsmedizin

\section{Korrespondenzadresse}

Prof. Dr. K. Püschel

Institut für Rechtsmedizin, Universi-

tätsklinikum Hamburg-Eppendorf

Butenfeld 34, 22529 Hamburg

pueschel@uke.de

\section{Literatur}

1. Püschel K, Lach H, Pollak S (2004) Die offiziellen Publikationsorgane der „Deutschen Gesellschaft für Rechtsmedizin" und ihre Vorgänger bzw. Nachfolger. In: Madea B, Bratzke H, Pollak S et al (Hrsg) 100 Jahre Deutsche Gesellschaft für Gerichtliche Medizin/Rechtsmedizin Vom Gründungsbeschluss 1904 zur Rechtsmedizin des 21. Jahrhunderts. Eigendruck DGRM, S. 654-672 